\title{
ON HOMOGENEOUS BIQUADRATIC DIOPHANTINEEQUATION:
}

$$
x^{4}-y^{4}=17\left(z^{2}-w^{2}\right) R^{2}
$$

\section{P.Jayakumar ${ }^{1}$, R.Venkatraman ${ }^{2}$}

\author{
${ }^{1}$ Professor of Mathematics, Periyar Maniammai University, Vallam,Thanajvur- 613403,Tamil Nadu, India \\ ${ }^{2}$ Ph.D. Scholar, Assistant Professor of Mathematics, SRM University, Vadapalani Campus, Chennai-600026. \\ Tamil Nadu, India.
}

\begin{abstract}
Five different methods of the non-zero non-negative solutions of non-homogeneous cubic Diophantine equation $x^{4}-y^{4}=17\left(z^{2}-\right.$ $\left.w^{2}\right) R^{2}$ are obtained. Some interesting relations among the special numbers and the solutions are exposed.
\end{abstract}

Keywords: The Method of Factorization, Integer Solutions, Linear Transformation, Relations and Special Numbers $* * *$

2010 Mathematics Subject Classification: 11D25

Symbols used:

$\mathrm{t}_{\mathrm{m}, \mathrm{n}}=\frac{1}{2} n[(m-2)-(m-4)]$

$\mathrm{p}_{\mathrm{n}}{ }^{\mathrm{m}}=\frac{1}{6}\left[3 n^{2}+n^{3}(m-2)-n(m-5)\right]$

$\mathrm{G}_{\mathrm{n}}=2 \mathrm{n}-1$

$\mathrm{Ct}_{16, n}=8 \mathrm{n}(\mathrm{n}+1)+1$

$\mathrm{OH}_{\mathrm{n}}=1 / 3 \mathrm{n}\left(2 \mathrm{n}^{2}+1\right)$

$\mathrm{SO}_{\mathrm{n}}=\mathrm{n}\left(2 \mathrm{n}^{2}-1\right)$

$\mathrm{ky}_{\mathrm{n}}=(2 \mathrm{n}+1)^{2}-2 \mathrm{carl}_{\mathrm{n}}-$ carol number

\section{INTRODUCTION}

The Mathematics is the Queen of all sciences. In particular, the Number theory is the King of Mathematics. The Number theory, in particular Diophantine equations have a blend of interesting problems. Many greatest Mathematicians was fascinated by problems in Diophantine equations.

For a vide review, one may try to see [1-12]. In this work, we are observed a lot of infinitely the non -zero integer values of the cubic Diophantine equation. Some interesting relations among the special numbers and the solutions are found.

\section{DESCRIPTION OF METHOD}

Let us consider the cubic Diophantine equation

$x^{4}-y^{4}=17\left(z^{2}-w^{2}\right) R^{2}$

Consider the transformations

$x=\mathrm{u}+\mathrm{v}, \mathrm{y}=\mathrm{u}-\mathrm{v}, \mathrm{z}=2 \mathrm{uv}+1, \mathrm{w}=2 \mathrm{uv}-1$

Using (2) in (1), it gives us the equation $u^{2}+v^{2}=17 R^{2}$

\subsection{Method: I}

We can write 17 as $17=(4+\mathrm{i})(4-\mathrm{i})$

and $\mathrm{R}=\mathrm{a}^{2}+\mathrm{b}^{2}=(\mathrm{a}+\mathrm{ib})(\mathrm{a}-\mathrm{ib})$

Using (4) and (5) in (3) and applying the process of factorization, it takes form as

$(\mathrm{u}+\mathrm{iv})(\mathrm{u}-\mathrm{iv})=(4+\mathrm{i})(4-\mathrm{i})(\mathrm{a}+\mathrm{ib})^{2}(\mathrm{a}-\mathrm{ib})$

The above equations give us

$(u+i v)=(4+i)(a+i b)^{2}$

$(\mathrm{u}+\mathrm{i} v)=(4-\mathrm{i})(\mathrm{a}-\mathrm{i} \mathrm{b})$

Comparing both sides of above equations, we obtain

$\mathrm{u}=\mathrm{u}(\mathrm{a}, \mathrm{b})=4 \mathrm{a}^{2}-4 \mathrm{~b}^{2}-2 \mathrm{ab}$

$\mathrm{v}=\mathrm{v}(\mathrm{a}, \mathrm{b})=\mathrm{a}^{2}-\mathrm{b}^{2}+8 \mathrm{ab}$

Using the values of $u$ and $v$ in (2) we get the non-zero integer values of $\mathrm{x}, \mathrm{y}, \mathrm{z}$ and $\mathrm{w}$ and $\mathrm{R}$ of (1) are furnished by

$x=x(a, b)=5 a^{2}-5 b^{2}+6 a b$

$y=y(a, b)=3 a^{2}-3 b^{2}-10 a b$

$z=z(a, b)=2\left(4 a^{4}+4 b^{4}-24 a^{2} b^{2}+30 a^{3} b-30 a b^{3}\right)+1$

$w=w(a, b)=2\left(4 a^{4}+4 b^{4}-24 a^{2} b^{2}+30 a^{3} b-30 a b^{3}\right)-1$

$\mathrm{R}=\mathrm{R}(\mathrm{a}, \mathrm{b})=\mathrm{a}^{2}+\mathrm{b}^{2}$

\section{Observations:}

$1.3 \mathrm{x}[a(\mathrm{a}+1), 1]-5 \mathrm{y}[\mathrm{a}(\mathrm{a}+1), 1]-68 \mathrm{P}_{\mathrm{a}}=0$

$2.3 \mathrm{x}[a(\mathrm{a}-1), 1]-5 \mathrm{y}[\mathrm{a}(\mathrm{a}-1), 1]-68 \mathrm{t}_{4, \mathrm{a}} \equiv 0(\operatorname{Mod} 2)$

$3 . z(\mathrm{~b}, 1)-\mathrm{w}(\mathrm{b}, 1) \equiv 0(\operatorname{Mod} 2)$

4.R(a+1, a+1) $-2 \mathrm{t}_{4, \mathrm{a}}-\mathrm{G}_{2 \mathrm{a}} \equiv 0(\operatorname{Mod} 3)$ 
$5 \cdot x(2, a)+y(2, a)+8 P_{a} \equiv 0(\operatorname{Mod} 2)$

6. $x(2,2)+y(2,2)+T_{16,2}=0$

7. $\left[A\left(2 A^{2}-1\right), 1\right]-5\left(S O_{A}\right)^{2}-6 S O_{A}+5 T_{5,2}=0$

$8.6 x(1,0)$ is a nastynumber.

9. $\mathrm{R}(2,3)-\mathrm{S}_{2}=0$

$10.3 x\left[2 \mathrm{a}^{2}+1, \mathrm{a}\right]-5 \mathrm{y}\left[2 \mathrm{a}^{2}+1, \mathrm{a}\right]-204 \mathrm{OH}_{\mathrm{a}}=0$

\subsection{Method: II}

We can also take17 as $17=(1+4 \mathrm{i})(1-4 \mathrm{i})$

Using (5) and (6) in equation (3) it takes form as

$(\mathrm{u}+\mathrm{i} v)(\mathrm{u}-\mathrm{i} \mathrm{v})=(1+4 \mathrm{i})(1-4 \mathrm{i})(\mathrm{a}+\mathrm{i} \mathrm{b})^{2}(\mathrm{a}-\mathrm{i} \mathrm{b})^{2}$

It gives as

$(u+i v)=(1+4 i)(a+i b)^{2}$

$(\mathrm{u}-\mathrm{iv})=(1-4 \mathrm{i})(\mathrm{a}-\mathrm{i} \mathrm{b})^{2}$

This leads to

$u=\mathrm{u}(\mathrm{a}, \mathrm{b})=\mathrm{a}^{2}-\mathrm{b}^{2}-8 \mathrm{ab}$

$v=\mathrm{v}(\mathrm{a}, \mathrm{b})=4 \mathrm{a}^{2}-4 \mathrm{~b}^{2}+2 \mathrm{ab}$

Putting the values of $u$ and $v$ in (2), the non-zero different values of $\mathrm{x}, \mathrm{y}, \mathrm{z}, \mathrm{w}$ and $\mathrm{R}$ of (1) are found as

$x=x(a, b)=5 a^{2}-5 b^{2}-6 a b$

$y=y(a, b)=-3 a^{2}+3 b^{2}-10 a b$

$z=z(a, b)=2\left(4 a^{4}+4 b^{4}-24 a^{2} b^{2}-30 a^{3} b+30 a b^{3}\right)+1$

$w=w(a, b)=2\left(4 a^{4}+4 b^{4}-24 a^{2} b^{2}-30 a^{3} b+30 a b^{3}\right)-1$

$\mathrm{R}=\mathrm{R}(\mathrm{a}, \mathrm{b})=\mathrm{a}^{2}+\mathrm{b}^{2}$

\section{Observations:}

1. $3 x\left[(2 a-1)^{2}, 1\right]+5 y\left[(2 a-1)^{2}, 1\right]+68\left(G_{a}\right)^{2}=0$

2. $\mathrm{y}(1,1)+\mathrm{R}(1,1)+\mathrm{OH}_{2} \equiv 0(\bmod 2)$

3. $3 x\left[a, 2 \mathrm{a}^{2}-1\right]+5 \mathrm{y}\left[2 \mathrm{a}^{2}-1\right]+68 \mathrm{So}_{\mathrm{a}}=0$

4. $\mathrm{z}(\mathrm{a}, 1)-\mathrm{W}(\mathrm{a}, 1)-\mathrm{KY}_{1} \equiv 0(\bmod 5)$

5. $\mathrm{z}(1, \mathrm{a})-\mathrm{W}(1, \mathrm{a})-\mathrm{Carl}_{2} \equiv 1(\bmod 2)$

6. $\mathrm{z}(1, \mathrm{a})-\mathrm{W}(1, \mathrm{a})-\mathrm{Carl}_{2}-\mathrm{TK}_{1}=0$

7. $x(a, a)-y(a, a)+16 T_{4, a}=0$

8.. $x(3,3)+y(3,3)+R(3,3)+T_{10,6}=0$

9. $y(1,2 a+1)-C t_{16, a}-S a+10 P_{a}-8 T_{4, a}=\mathrm{s}^{3}$, where $\mathrm{s}$ is an integer

10. $\mathrm{x}(\mathrm{b}, \mathrm{b}+1)+5 \mathrm{G}_{2}, b+6 P_{b}$ is a $n$ even int eger.

$11 . z(2,2)+w(2,2) \equiv 0(\bmod 2)$

\subsection{Method: III}

(3) can also be written as $1 * \mathrm{u}^{2}=17 \mathrm{R}^{2}-v^{2}$

Take as $u=17 a^{2}-b^{2}=(\sqrt{17} a+b)(\sqrt{17} a-b)$
Take 1 as $1=(\sqrt{17}+4)(\sqrt{17}-4)$

Using (8) and (9) in (7), it takes the form as

$(\sqrt{17}+4)(\sqrt{17}-4)(\sqrt{17} a+b)^{2}(\sqrt{17} a-b)^{2}=$

$(\sqrt{17} \mathrm{R}+\mathrm{v})(\sqrt{17} \mathrm{R}-\mathrm{v})$

This gives us

$(\sqrt{17}+4)(\sqrt{17} \mathrm{a}+\mathrm{b})^{2}=(\sqrt{17} \mathrm{R}+\mathrm{v})$

$(\sqrt{17}-4)(\sqrt{17} \mathrm{a}-\mathrm{b})^{2}=(\sqrt{17} \mathrm{R}-\mathrm{v})$

This found as

$\mathrm{R}=\mathrm{R}(\mathrm{a}, \mathrm{b})=17 \mathrm{a}^{2}+\mathrm{b}^{2}+8 \mathrm{ab}$

$\mathrm{v}=\mathrm{v}(\mathrm{a}, \mathrm{b})=68 \mathrm{a}^{2}+4 \mathrm{~b}^{2}+34 \mathrm{ab}$

Putting the values of $u$ and $v$ in (2), the non - zero different values of $\mathrm{x}, \mathrm{y}, \mathrm{z}, \mathrm{R}$ and $\mathrm{w}$ of (1) are determined as

$\mathrm{x}=\mathrm{x}(\mathrm{a}, \mathrm{b})=85 \mathrm{a}^{2}+3 \mathrm{~b}^{2}+34 \mathrm{ab}$

$\mathrm{y}=\mathrm{y}(\mathrm{a}, \mathrm{b})=-51 \mathrm{a}^{2}-5 \mathrm{~b}^{2}-34 \mathrm{ab}$

$z=z(a, b)=2\left(1156 a^{4}-4 b^{4}-34 a b^{3}+578 a^{3} b\right)+1$

$w=w(a, b)=2\left(1156 a^{4}-4 b^{4}-34 a b^{3}+578 a^{3} b\right)-1$

$\mathrm{R}=\mathrm{R}(\mathrm{a}, \mathrm{b})=17 \mathrm{a}^{2}+\mathrm{b}^{2}+8 \mathrm{ab}$

\section{Observations:}

1. $R(3 a, 3 a)-234 t_{4, a}=0$

2. $5 x(a, 1)-3 y(a, 1)-C_{16, a}-S a-564 t_{4, a}+G_{35 a} \equiv 0(\bmod 3)$

3. $\mathrm{R}(\mathrm{a}, 2 \mathrm{a}-1)-\mathrm{Sa}-\mathrm{Ct}_{16, \mathrm{a}}-23 \mathrm{t}_{4, \mathrm{a}}+\mathrm{G}_{3 \mathrm{n}} \equiv 0(\bmod 2)$

4. $3 \mathrm{Z}(1,1)-3 \mathrm{~W}(1,1)-\mathrm{OH}_{2}=0$

5. $x\left[a, a\left(2 a^{2}-1\right)\right]+y\left[a, a\left(2 a^{2}-1\right)\right]-34 t_{4, a}+2 S_{a}=0$

6. $y(1,1)-P_{8}=0$.

7. $5 x[A,(A+1)(2 A+1)]-3 y[A,(A+1)(2 A+1)]-578 T_{4, A}=0$.

8. $x(1, A)+y(1, A)+2 T_{4, A}-T_{7,4}=0$.

9..R(2,2) $\equiv 0(\bmod 2)$.

\subsection{Pattern: IV}

Again take (3) as $1 * \mathrm{v}^{2}=17 \mathrm{R}^{2}-\mathrm{u}^{2}$

Take 1 as $1=\frac{(\sqrt{17}+1)(\sqrt{17}-1)}{16}$

Put $v=17 \mathrm{a}^{2}-\mathrm{b}^{2}=(\sqrt{17} \mathrm{a}-\mathrm{b})(\sqrt{17} \mathrm{a}+\mathrm{b})$

Using (12) and (13) in (11), it takes form as

$\frac{(\sqrt{17}+1)(\sqrt{17}-1)}{16}(\sqrt{17} a-b)^{2}(\sqrt{17} a+b)^{2}=(\sqrt{17} R-u)$

$(\sqrt{17} \mathrm{R}+\mathrm{u})$

This gives us

$\mathrm{R}=\mathrm{R}(\mathrm{a}, \mathrm{b})=\frac{1}{4}\left(17 \mathrm{a}^{2}+\mathrm{b}^{2}+2 \mathrm{ab}\right)$

$\mathrm{u}=\mathrm{u}(\mathrm{a}, \mathrm{b})=\frac{1}{4}\left(17 \mathrm{a}^{2}+\mathrm{b}^{2}+34 \mathrm{ab}\right)$ 
Putting ' $\mathrm{a}$ ' by $4 \mathrm{~A}$ and ' $\mathrm{b}$ ' by $4 \mathrm{~B}$ in the above

equations (13) and (15), it is found as

$\mathrm{R}=\mathrm{R}(\mathrm{A}, \mathrm{B})=68 \mathrm{~A}^{2}+4 \mathrm{~B}^{2}+8 \mathrm{AB}$

$\mathrm{u}=\mathrm{u}(\mathrm{A}, \mathrm{B})=68 \mathrm{~A}^{2}+4 \mathrm{~B}^{2}+136 \mathrm{AB}$

$\mathrm{v}=\mathrm{v}(\mathrm{A}, \mathrm{B})=272 \mathrm{~A}^{2}-16 \mathrm{~B}^{2}$

On putting the values of $\mathrm{u}$ and $\mathrm{v}$ in (2), the non -zero different integrals values of $\mathrm{x}, \mathrm{y}, \mathrm{z}, \mathrm{w}$ and $\mathrm{R}$ of (1) are found as

$\mathrm{x}=\mathrm{x}(\mathrm{A}, \mathrm{B})=340 \mathrm{~A}^{2}-12 \mathrm{~B}^{2}+136 \mathrm{AB}$

$\mathrm{y}=\mathrm{y}(\mathrm{A}, \mathrm{B})=-204 \mathrm{~A}^{2}+20 \mathrm{~B}^{2}+136 \mathrm{AB}$

$\mathrm{z}=\mathrm{z}(\mathrm{A}, \mathrm{B})=2\left(18496 \mathrm{~A}^{4}-64 \mathrm{~B}^{4}+36992 \mathrm{~A}^{3} \mathrm{~B}-\right.$

$\left.2176 \mathrm{AB}^{3}\right)+1$

$w=w(A, B)=2\left(18496 A^{4}-64 B^{4}+36992 A^{3} B-\right.$

$\left.2176 \mathrm{AB}^{3}\right)-1$

$\mathrm{R}=\mathrm{R}(\mathrm{A}, \mathrm{B})=68 \mathrm{~A}^{2}+4 \mathrm{~B}^{2}+8 \mathrm{AB}$

\section{Observations:}

1. $R(2 b, 2 b)-320 t_{4, b}=0$

2. $x(b+1, b+2)-y(b+1, b+2)-512 t_{4, b}-G_{480 b}+P_{6}=0$

3. $\mathrm{y}\left[1, \mathrm{~A}\left(2 \mathrm{~A}^{2}-1\right)\right]+3\left[1, \mathrm{~A}\left(2 \mathrm{~A}^{2}-1\right)\right]-32\left(\mathrm{SO}_{\mathrm{A}}\right)^{2}-160 \mathrm{So}_{\mathrm{A}}=0$

4. $\mathrm{x}(1, \mathrm{~A})-\mathrm{y}(1, \mathrm{~A})+32 \mathrm{~T}_{4, \mathrm{~A}}-\mathrm{S}_{10}=0(\bmod 3)$

5. $3 \mathrm{z}(4,1)-3 \mathrm{~W}(\mathrm{~A}, 1)$ is a Nasty number

$6.12 y\left[1, B\left(2 B^{2}+1\right)\right]+20 x\left[1, B\left(2 B^{2}+1\right)\right]-4352 S O_{B} \equiv 0(\bmod 2)$.

7. $x(2,2)-y(2,2) . \equiv 0(\bmod 2)$.

8. $x(A, 0)-340 t_{4, A}=0$

9. $x[1, A(A+1)]-y[1, A(A+1)]+32\left(P_{A}\right)^{2} \equiv 0(\bmod 2)$.

10. $z(2,2)+w(2,2) \equiv 0(\bmod 2)$.

\subsection{Pattern: V}

Let us take (3) as $\mathrm{u}^{2}-\mathrm{R}^{2}=16 \mathrm{R}^{2}-\mathrm{v}^{2}$

$(\mathrm{u}+\mathrm{R})(\mathrm{u}-\mathrm{R})=(4 \mathrm{R}+\mathrm{v})(4 \mathrm{R}-\mathrm{v})$.

$\frac{u+R}{4 R+V}=\frac{4 R-V}{U-R}=\frac{A}{B}, \mathrm{~B} \neq 0$

This gives us the equations as

$-\mathrm{uA}+\mathrm{R}(4 \mathrm{~B}+\mathrm{A})-\mathrm{VB}=0$

$\mathrm{uB}+\mathrm{R}(\mathrm{B}-4 \mathrm{~A})-\mathrm{VA}=0$

By cross multiplication, it leads to

$\mathrm{u}=\mathrm{u}(\mathrm{A}, \mathrm{B})=-\mathrm{A}^{2}-\mathrm{B}^{2}-8 \mathrm{AB}$

$\mathrm{R}=\mathrm{R}(\mathrm{A}, \mathrm{B})=-\mathrm{A}^{2}-\mathrm{B}^{2}$

$\mathrm{v}=\mathrm{v}(\mathrm{A}, \mathrm{B})=4 \mathrm{~A}^{2}-4 \mathrm{~B}^{2}-2 \mathrm{AB}$

Puting the values of $u$ and $v$ in (2), the non - zero different integral values of $\mathrm{x}, \mathrm{y}, \mathrm{z}, \mathrm{w}$ and $\mathrm{R}$ of (1) are found as,

$$
\begin{aligned}
& x=x(A, B)=3 A^{2}-6 B^{2}-10 A B \\
& y=y(A, B)=-5 A^{2}+5 B^{2}-6 A B \\
& z=z(A, B)=2\left[-4 A^{4}-4 B^{4}+8 A^{2} B^{2}-30 A^{3} B+30 A B^{3}\right]+1 \\
& w=w(A, B)=2\left[-4 A^{4}-4 B^{4}+8 A^{2} B^{2}-30 A^{3} B+30 A B^{3}\right]-1 \\
& R=R(A, B)=-A^{2}-B^{2}
\end{aligned}
$$

\section{Observations:}

1. $\mathrm{R}[1, \mathrm{~A}(\mathrm{~A}+1)]+\left(\mathrm{P}_{\mathrm{A}}\right)^{2}=$ woodall number

2. $\mathrm{R}[\mathrm{A}+1, \mathrm{~A}+2]+\mathrm{P}_{\mathrm{A}} \equiv 0(\bmod 3)$

3. $5 x\left[1, A\left(2 \mathrm{~A}^{2}-1\right)\right]+3\left[1, \mathrm{~A}\left(2 \mathrm{~A}^{2}-1\right)\right]+68 \mathrm{SO}_{\mathrm{A}}=0$

4. $\mathrm{x}[1, \mathrm{~A}(\mathrm{~A}+1)]-3 \mathrm{R}[1, \mathrm{~A}(\mathrm{~A}+1)]+10 \mathrm{P}_{\mathrm{A}}=6$,

a Nasty Number

5. $\mathrm{y}(2 \mathrm{~A}, 1)+5 \mathrm{R}(2 \mathrm{~A}, 1)+28 \mathrm{t}_{4, \mathrm{~A}}+12 \mathrm{P}_{\mathrm{A}}=0$

$6.6 x\left[A\left(2 A^{2}+1\right), 1\right]+10 y\left[A\left(2 A^{2}+1\right), 1\right]-360 O H_{A}=0$.

7. $x(2,2)-y(2,2) . i s$. is a perfectsquare.

8. $z[2,2]-w(2,2)$. is a square number

9. $R[A,(2 A-1)]+T_{12, A}=$ carol number

10. $x\left[A\left(2 A^{2}-1\right), A\left(2 A^{2}-1\right)\right]+10 S O_{A}=0$

\section{CONCLUSION}

It is interest to see that in (2), the transformations for $\mathrm{z}$ and $\mathrm{w}$ maybe taken as $\mathrm{z}=2 \mathrm{u}+\mathrm{v}$ and $\mathrm{w}=2 \mathrm{u}-\mathrm{v}$. For this choice, the values of $x, y$ and $R$ are the same as above where as the valueof $\mathrm{z}$ and $\mathrm{w}$ changes for every method. One may try to see biquadratic Diophantine equations with multivariables $(\geq 5)$ and search for their non-zero distinct integer solutions together with their similar observations.

\section{REFERENCES}

[1]. Dickson, L.E., History of theory of numbers, Vol.11, Chelsea publishing company, New -York (1952).

[2]. Mordell, L.J., Diophantine equation, Academic press, London (1969) Journal of Science and Research, Vol(3) Issue 12, 20-22 (December -14)

[3]. Jayakumar. P, Sangeetha, K "Lattice points on the cone $x^{2}+9 y^{2}=50 z^{2}$ " International Journal of Science and Research, Vol (3), Issue 12, 20-22 December2014)

[4]. Jayakumar P, Kanaga Dhurga, C," OnQuadratic Diopphantine equation $x^{2}+16 y^{2}=20 z^{2,}$ Galois J. Maths, 1(1) (2014), 17-23.

[5]. Jayakumar. P, Kanaga Dhurga. C, "Lattice points onthecone $\mathrm{x}^{2}+9 \mathrm{y}^{2}=50 \mathrm{z}^{2}$ " Diophantus J. Math,3(2) (2014), 61-71

[6]. Jayakumar. P, Prabha. S "On Ternary Quadratic Diophantine equation $\mathrm{x}^{2}+15 \mathrm{y}^{2}=14 \mathrm{z}^{2}$ " Archimedes J. Math., 4(3) (2014), 159-164.

[7]. Jayakumar, P, Meena, J "Integral solutions of the Ternary Quadratic Diophantine equation: $x^{2}+7 y^{2}=$ $16 \mathrm{z}^{2}$ International Journal of Science and Technology, Vol.4, Issue 4, 1-4, Dec 2014.

[8]. Jayakumar. P, Shankarakalidoss, G "Latticepoints on

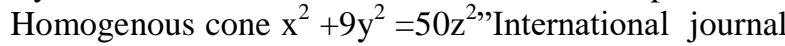
of Science and Research, Vol (4), Issue 1, 2053-2055, January -2015 . 
[9]. Jayakumar. P, Shankarakalidoss. G "Integral points on the Homogenous cone $x^{2}+y^{2}=10 z^{2}$ International Journalfor Scienctific Research and Development, Vol (2), Issue 11, 234-235, January -2015

[10].Jayakumar.P, Prabha.S "Integral points on the cone $x^{2}$ $+25 \mathrm{y}^{2}=17 \mathrm{z}^{2}$, International Journal of Science andResearch Vol(4), Issue 1, 2050-2052, January-2015.

[11].Jayakumar.P, Prabha. S, "Lattice points on the cone $x^{2}$ $+9 y^{2}=26 z^{2}$ "International Journal of Science and Research Vol (4), Issue 1, 2050-2052, January -2015

[12].Jayakumar. P, Sangeetha. K, "Integral solutionofthe Homogeneous Biquadratic Diophantine equation with six unknowns: $\left(\mathrm{x}^{3}-\mathrm{y}^{3}\right) \quad \mathrm{z}=\left(\mathrm{W}^{2}-\mathrm{P}^{2}\right) \mathrm{R}^{4 \text { c International }}$ Journal of Science and Research, $\operatorname{Vol}(3)$, Issue 12, December-2014)

\section{BIOGRAPHY}

1. Dr. P. Jayakumar received the B. Sc, M.Sc degrees in Mathematics from Madras University in 1980 and 1983 and the M. Phil, Ph.D degrees in Mathematics from Bharathidasan University, Thiruchirappalli in 1988 and 2010.Who is now working as Professor of Mathematics, Periyar Maniammai University, Vallam, Thanajvur-613 403,Tamil Nadu,India.

2. R.Venkatraman received the B.Sc, M.Sc, and MPhil degrees in Mathematics from Bharathidasan University, Thiruchirappalli in 2002, 2004 and 2006. Who is now working as Assistant Professor of Mathematics, SRM UniversityVadapalani Campus, and Chennai-600026? 